\title{
On Stability of Periodic Solutions of Lienard Type Equations
}

\author{
Zijian Yin and Hongbin Chen \\ School of Mathematics and Statistics, Xian Jiaotong University, Xian 710049, China \\ Correspondence should be addressed to Zijian Yin; zijianyin@126.com
}

Received 10 September 2017; Accepted 10 October 2017; Published 13 November 2017

Academic Editor: Douglas R. Anderson

Copyright (c) 2017 Zijian Yin and Hongbin Chen. This is an open access article distributed under the Creative Commons Attribution License, which permits unrestricted use, distribution, and reproduction in any medium, provided the original work is properly cited.

We use the Floquet theory to analyze the stability of periodic solutions of Lienard type equations under the asymptotic linear growth of restoring force in this paper. We find that the existence and the stability of periodic solutions are determined primarily by asymptotic behavior of damping term. For special type of Lienard equation, the uniqueness and stability of periodic solutions are obtained. Furthermore, the sharp rate of exponential decay of the stable periodic solutions is determined under suitable conditions imposed on restoring force.

\section{Introduction}

This paper is devoted to the stability of large periodic solutions of the following Lienard type equation:

$$
x^{\prime \prime}(t)+\frac{d}{d t} f(t, x(t))+g(x(t))=h(t),
$$

where $(d / d t) f(t, x(t))=f_{t}(t, x(t))+f_{x}(t, x(t)) x^{\prime}(t), g(x)$ is a continuous function and $h(t)$ is a $T$-periodic function. The existence and multiplicity of periodic solutions of (1) or more general types of nonlinear second-order differential equations

$$
x^{\prime \prime}(t)+\frac{d}{d t} f(t, x(t))+g(t, x(t))=h(t)
$$

have been investigated extensively by many authors. For details, we refer the reader to [1-4]. One can mention, for example, the papers by Fonda and Habets [5] or more recent papers by Qian [6] and the literature therein. In these papers, the asymptotic behavior of restoring force and damping term are controlled by the inequalities

$$
\begin{aligned}
& a(t) \leq \liminf _{|x| \rightarrow \infty} \frac{g(t, x)}{x} \leq \limsup _{|x| \rightarrow \infty} \frac{g(t, x)}{x} \leq b(t), \\
& c(t) \leq \liminf _{|x| \rightarrow \infty} \frac{f(t, x)}{x} \leq \underset{|x| \rightarrow \infty}{\lim \sup } \frac{f(t, x)}{x} \leq d(t) .
\end{aligned}
$$

These tend to keep away the combinational effects of the quotients $g(t, x) / x$ and $f(t, x) / x$ from the spectrum of the linear operator $L x=-x^{\prime \prime}$ as $|x| \rightarrow \infty$. However, the sign condition on damping term on solvability of (1) seems to be ignored. The aim of the paper is to show that sign condition plays dominate role on the existence of periodic solutions of (1) under the condition that $g$ is asymptotically linear. Resonance phenomenon may appear when $\lim _{x \rightarrow \infty}(f(t, x) / x) \equiv 0$. In this case, the Landesman-Lazer type solvability condition should be imposed. In this paper, the following hypotheses on $f$ and $g$ are imposed:

$$
\begin{gathered}
\lim _{x \rightarrow \infty} \frac{g(x)}{x}=c \neq 0, \\
\lim _{x \rightarrow \infty} \frac{f(t, x)}{x}=a(t) \gg 0
\end{gathered}
$$

or

$$
\begin{aligned}
\lim _{x \rightarrow \infty} \frac{g(x)}{x} & =c \neq\left(\frac{2 n \pi}{T}\right)^{2}, \\
\lim _{x \rightarrow \infty} \frac{f(t, x)}{x} & \equiv 0,
\end{aligned}
$$

where the second limit converges uniformly for $t \in[0, T]$. 
When the hypotheses

$$
\begin{aligned}
\lim _{x \rightarrow \infty} \frac{f(t, x)}{x} & \equiv 0, \\
c & =\left(\frac{2 n \pi}{T}\right)^{2},
\end{aligned}
$$

are satisfied, which can be treated as the resonance conditions, (1) does not exist with $T$-periodic solution in general, even if $f_{x}(t, x)$ does not change sign for all $x \in \mathbb{R}$. A counterexample will be given at the end of Section 2. In this case, an additional condition should be imposed to guarantee the existence.

The related results about the stability of periodic solutions were less extensively studied. In [7], Lazer and Mckenna established stability results by converting the equation to a fixed point problem. Recently, more complete results concerning the stability and the sharpness of the rate of decay of periodic solutions were obtained by Chen and $\mathrm{Li}$ in $[8,9]$.

The following notations will be used throughout the rest of the paper:

(1) $L_{T}^{p}: T$-periodic function $u \in L^{p}[0, T]$ with $\|u\|_{p}$ for $1 \leq p \leq \infty$

(2) $C_{T}^{k}: T$-periodic function $u \in C^{k}[0, T], k \geq 0$ with $C^{k}$-norm;

(3) $\alpha(t) \gg \beta(t)$ : if $\alpha(t) \geq \beta(t)$ on $[0, T]$ and $\alpha(t)>\beta(t)$ on some positive measure subset.

The main results of this paper are the following.

Theorem 1. Assume $g(x) \in C(\mathbb{R} \times \mathbb{R}), f(t, x) \in C^{1}(\mathbb{R} \times \mathbb{R})$, and T-periodic in t, satisfying (4) or (5). Then (1) has at least one $T$-periodic solution.

Let us introduce the following symbols:

$$
\begin{aligned}
\alpha_{ \pm}(t) & =\liminf _{x \rightarrow \pm \infty} q(t, x), \\
\beta_{ \pm}(t) & =\lim _{x \rightarrow \pm \infty} \sup q(t, x), \\
a(t) & =\lim _{x \rightarrow+\infty} f(t, x), \\
b(t) & =\lim _{x \rightarrow-\infty} f(t, x),
\end{aligned}
$$

where $a(t), b(t) \in C_{T}^{1}$.

Theorem 2. Assume that $q(t, x), f(t, x)$ is bounded, where $g(t, x)=(2 n \pi / T)^{2}+q(t, x)$ and (6). Then (2) has a T-periodic solution provided that either

$$
\begin{gathered}
\int_{0}^{T} h z d t+\int_{z>0} a^{\prime} z d t+\int_{z<0} b^{\prime} z d t \\
<\int_{z>0} \alpha_{+} z d t+\int_{z<0} \beta_{-} z d t
\end{gathered}
$$

or

$$
\begin{gathered}
\int_{0}^{T} h z d t+\int_{z>0} a^{\prime} z d t+\int_{z<0} b^{\prime} z d t \\
<\int_{z<0} \alpha_{-} z d t+\int_{z>0} \beta_{+} z d t,
\end{gathered}
$$

for any $z(t) \in[\sin (2 n \pi / T) t, \cos (2 n \pi / T) t]$.

Remark 3. When $a(t)$ and $b(t)$ are continuous, the conclusion of Theorem 2 still holds if the second and third term in (8) or (9) are replaced by $-\left[\int_{z>0} a z^{\prime} d t+\int_{z<0} b z^{\prime} d t\right]$.

In particular, by Theorem 2, we see that the following well known Landesman-Lazer type solvability conditions hold, if both $a(t)$ and $b(t)$ are all constants.

Theorem 4. Under the same conditions of Theorem 2, where $a(t)$ and $b(t)$ are constants, then (1) has a T-periodic solution if either

$$
\int_{0}^{T} h z d t<\int_{z>0} \alpha_{+} z d t+\int_{z<0} \beta_{-} z d t
$$

or

$$
\int_{0}^{T} h z d t<\int_{z<0} \alpha_{-} z d t+\int_{z>0} \beta_{+} z d t .
$$

When $g(x)$ is a linear function, more refined results can be obtained. Concerning uniqueness and stability as well as the rate of decay to the unique periodic solution, we have the following.

Theorem 5. Assume that $g(x)=c x$ where $c>0$ and $f(t$, $x) \in C^{1}(\mathbb{R} \times \mathbb{R})$, such that

$$
\begin{aligned}
f_{x}^{\prime}(t, x(t)) & >0, \\
\lim _{n \rightarrow \infty} \frac{f(t, x)}{x} & =a(t) \gg 0 .
\end{aligned}
$$

Then

(1) (1) has a unique T-periodic solution;

(2) the unique T-periodic solution is locally exponential asymptotically stable;

(3) the unique T-periodic solution is globally asymptotically stable.

Furthermore, if the conditions,

$$
\begin{aligned}
& \frac{1}{2} f_{t x}^{\prime \prime}(t, x(t))+\frac{1}{2} f_{x x}^{\prime \prime}(t, x(t)) x^{\prime}(t)-\frac{1}{4} f_{x}^{\prime 2}(t, x(t)) \\
& +c \gg \frac{n^{2} \pi^{2}}{T^{2}}, \\
& \frac{1}{2} f_{t x}^{\prime \prime}(t, x(t))+\frac{1}{2} f_{x x}^{\prime \prime}(t, x(t)) x^{\prime}(t)-\frac{1}{4} f_{x}^{\prime 2}(t, x(t)) \\
& \quad+c \ll \frac{(n+1)^{2} \pi^{2}}{T^{2}},
\end{aligned}
$$


hold for some $n \geq 1$, then every solution to (1) that is near the unique $T$-periodic solution decays uniformly at the exponential rate of $(1 / 2) \int_{0}^{T} f_{x}^{\prime}\left(t, x_{0}(t)\right) d t$.

\section{Preliminaries}

In this section we shall recall some basic results about topological methods.

Consider the periodic boundary value problem

$$
\begin{aligned}
x^{\prime} & =F(t, x), \\
x(0) & =x(T),
\end{aligned}
$$

where $F:[0, T] \times \mathbb{R}^{n} \rightarrow \mathbb{R}^{n}$ is a continuous function and $T$ periodic in $t$. In order to use a homotopic method to compute the degree, we assume that $h:[0, T] \times \mathbb{R}^{n} \times[0,1] \rightarrow \mathbb{R}^{n}$ is a continuous function such that

$$
\begin{aligned}
& h(t, x, 1)=F(t, x), \\
& h(t, x, 0)=G(x),
\end{aligned}
$$

where $G(x)$ is continuous. The following continuation theorem is due to Mawhin [10].

Lemma 6. Let $\Omega \subset C_{T}$ be an open bounded set such that the following conditions are satisfied.

(1) There is no $x \in \partial \Omega$ such that $x^{\prime}(t)=h(t, x, \lambda), \forall \lambda \in$ $[0,1)$.

(2) $\operatorname{deg}\left(G, \Omega \cap \mathbb{R}^{n}, 0\right) \neq 0$.

Then (12) has at least one solution.

Next, we consider the homogeneous periodic equation

$$
x^{\prime \prime}(t)+(\alpha(t) x(t))^{\prime}+c x(t)=0,
$$

where $c \neq 0$ is constant and $\alpha(t) \in L_{T}^{\infty}$.

The following Lemma is crucial to the argument for existence of periodic solutions.

Lemma 7. Assume that $\alpha(t) \in L_{T}^{\infty}$ satisfies either $\alpha(t) \gg 0$ or $\alpha(t) \ll 0$. Then (14) does not admit any nontrivial T-periodic solutions.

Proof. Suppose on the contrary that there exists a nontrivial $T$-periodic solution $x(t)$. Multiplying both sides of (14) by $x^{\prime}(t)$ and $\alpha(t) x(t)$, respectively, integrating by parts, and applying the boundary condition, we get

$$
\begin{array}{r}
\int_{0}^{T} x^{\prime \prime}(t) \alpha(t) x(t) d t=0, \\
\int_{0}^{T} x^{\prime \prime}(t) \alpha(t) x(t) d t+c \int_{0}^{T} \alpha(t) x^{2}(t) d t=0 ;
\end{array}
$$

then

$$
c \int_{0}^{T} \alpha(t) x^{2}(t) d t=0
$$

This implies we have $x(t) \equiv 0$ on positive measure subset of $[0, T]$. It follows from Rolle's theorem that the derivative $x^{\prime}(t)$ has a zero, between two zeros of $x(t)$. Let $t_{0}$ be an accumulation point of zero of $x(t)$, such that $t_{0}$ exists. Otherwise, the zeros of $x(t)$ are isolated; hence the set consisting of zeros of $x(t)$ is a zero measure set. Evidently, at such point $x\left(t_{0}\right)=$ $x^{\prime}\left(t_{0}\right)=0$. According to a theorem concerning the uniqueness of initial value problem, we have $x(t) \equiv 0$ on $[0, T]$.

Let us give a counterexample, which demonstrates that (1) does not possess any periodic solutions under condition (6).

Consider the following equation:

$$
x^{\prime \prime}+\frac{1}{1+x^{2}} x^{\prime}+n^{2} x=a \cos n t
$$

where $f(t, x)=\arctan x$ and $T=2 \pi$ satisfies condition (6) but (17) does not admit any $2 \pi$-periodic solutions for $|a|>$ $n \pi$.

Indeed, if $x(t)$ is a $2 \pi$-periodic solution of (17), multiplying both sides of (17) by cos $n t$ and integrating over a period, we obtain

$$
\begin{aligned}
a \pi & =\int_{0}^{2 \pi} a \cos ^{2} n t d t=\int_{0}^{2 \pi} \frac{\cos n t}{1+x^{2}} x^{\prime} d t \\
& =n \int_{0}^{2 \pi} \sin n t \arctan x d t,
\end{aligned}
$$

which means that

$$
|a| \pi<2 \pi n \times 1 \times \frac{\pi}{2}=\pi^{2} n .
$$

Thus, the equation does not have any $2 \pi$-periodic solutions for $|a|>n \pi$. Moreover, according to Massera's theorem [11], we obtain that any solutions of (17) are unbounded for $|a|>n \pi$.

For convenience, we begin with a definition.

Definition 8. Let $p(t)$ be a $T$-periodic solution of $x^{\prime}(t)=$ $f(x, t)$, where $f(x, t) \in C^{1}(\mathbb{R} \times \mathbb{R})$. Then $p$ is stable if for each $\varepsilon>0$ there exists a $\delta>0$, such that if $\psi(t)$ is any solution of $x^{\prime}(t)=f(x, t)$ for which

$$
\left|\psi\left(t_{0}\right)-p\left(t_{0}\right)\right|<\delta
$$

at some $t_{0}$, then

$$
|\psi(t)-p(t)|<\varepsilon
$$

for any $t \geq t_{0}$.

And next, we shall recall a principle of linearized stability for periodic systems.

Let $x_{0}$ be a $T$-periodic solution of (12); then we associate the $T$-periodic solution $x_{0}$ with the linearized equation

$$
y^{\prime}=F_{x}^{\prime}\left(t, x_{0}\right) y .
$$

Let $M(t)$ be the fundamental matrix of (22) and $\mu_{1}$ and $\mu_{2}$ the eigenvalues of the matrix $M(T)$. Then $x\left(t, x_{0}\right)$ is exponential asymptotically stable if and only if $\left|\mu_{i}\right|<1$, 
$i=1,2$. Otherwise, if there exists an eigenvalue of $M(T)$ with modulus greater than one, then $x\left(t, x_{0}\right)$ is unstable.

In order to show that every solution of the nonlinear equation (1) locally decays at the rate of $(1 / 2) \int_{0}^{T} f_{x}^{\prime}\left(t, x_{0}(t)\right) d t$ to the unique $T$-periodic solution, we need the following $C^{1}$ version of the Hartman-Grobman theorem [12].

Lemma 9. Let $U$ be an open neighborhood of 0 and $f: U \subset$ $\mathbb{R}^{n} \rightarrow \mathbb{R}^{n}$ be a $C^{1}$ function such that $f_{x}^{\prime}(0): \mathbb{R}^{n} \rightarrow \mathbb{R}^{n}$ is a contraction mapping. Then $f$ is $C^{1}$ conjugate equivalent to $f_{x}^{\prime}(0)$.

Consider the following boundary value problem:

$$
\begin{aligned}
x^{\prime \prime}(t)+q(t) x(t) & =0, \\
x(\xi) & =x(\xi+T)=0, \\
& \operatorname{sgn} x^{\prime}(\xi)=\operatorname{sgn} x^{\prime}(\xi+T) ;
\end{aligned}
$$

we have the following lemma which is given by the author in [9]

Lemma 10. Let $q(t) \in L^{\infty}(0, T)$ such that

$$
\frac{[2 n \pi]^{2}}{T^{2}} \ll q(t) \ll \frac{[2(n+1) \pi]^{2}}{T^{2}}
$$

for some $n \in \mathbb{N}$. Then (23) does not admit any nontrivial solution.

Proof. Consider the eigenvalue problem

$$
\begin{aligned}
x^{\prime \prime}(t)+\lambda x(t) & =0, \\
x(\xi) & =x(\xi+T)=0 ;
\end{aligned}
$$

we have $\lambda_{n}=(n \pi)^{2} / T^{2}$, the $n$th eigenvalue of the above equation. If $x$ is a nontrivial solution of (23), then $x$ is an eigenfunction associated with eigenvalue $\mu_{k}(q(t))=0$ for some $k$ of the eigenvalue problem

$$
\begin{aligned}
x^{\prime \prime}(t)+q(t) x(t)+\mu x(t) & =0, \\
x(\xi) & =x(\xi+T)=0 .
\end{aligned}
$$

Since $\lambda_{2 n} \ll q(t) \ll \lambda_{2(n+1)}$, it follows from the comparison principle that

$$
\begin{aligned}
\lambda_{k}-\lambda_{2(n+1)} & =\mu_{k}\left(\lambda_{2(n+1)}\right)<\mu_{k}(q(t))=0 \\
& <\mu_{k}\left(\lambda_{2 n}\right)=\lambda_{k}-\lambda_{2 n} .
\end{aligned}
$$

Thus $2 n<k<2(n+1), k=2 n+1$. On the other hand, it follows from Sturm theory that the eigenfunction corresponding to the eigenvalue $\mu_{2 n+1}$ of (26) has exact $2 n$ zeros in $(\xi, \xi+$ $T)$, which indicates that $\operatorname{sgn} x^{\prime}(\xi)=-\operatorname{sgn} x^{\prime}(\xi+T)$. Together with the boundary condition of (23), we have $x^{\prime}(\xi)=x^{\prime}(\xi+$ $T)=0$. This is a contradiction.

Now we consider the homogeneous periodic equation

$$
y^{\prime \prime}(t)+\frac{d}{d t}\left(f_{x}^{\prime}\left(t, x_{0}(t)\right) y(t)\right)+c y(t)=0 .
$$

We have the following.
Lemma 11. Assume that there exists an integer $n \in \mathbb{N}$ such that

$$
\begin{aligned}
& \frac{1}{2} f_{t x}^{\prime \prime}(t, x(t))+\frac{1}{2} f_{x x}^{\prime \prime}(t, x(t)) x^{\prime}(t)-\frac{1}{4} f_{x}^{\prime 2}(t, x(t)) \\
& \quad+c \gg \frac{[2 n \pi]^{2}}{T^{2}}, \\
& \frac{1}{2} f_{t x}^{\prime \prime}(t, x(t))+\frac{1}{2} f_{x x}^{\prime \prime}(t, x(t)) x^{\prime}(t)-\frac{1}{4} f_{x}^{\prime 2}(t, x(t)) \\
& \quad+c \ll \frac{[2(n+1) \pi]^{2}}{T^{2}} .
\end{aligned}
$$

Then (28) does not admit any positive Floquet multipliers. In particular, (28) does not admit any nontrivial $T$-periodic solutions.

Proof. Suppose that there is a nontrivial $T$-periodic solution of (28) such that $y(t+T)=\alpha y(t)$ with $\alpha>0$.

Set

$$
y(t)=\exp \left[\frac{-1}{2} \int_{0}^{t} f_{x}^{\prime}\left(s, x_{0}(s)\right) d s\right] u(t) ;
$$

then $u(t)$ solves the following equation:

$$
\begin{aligned}
u^{\prime \prime} & (t)+\left[c+\frac{1}{2} f_{x x}^{\prime \prime}\left(t, x_{0}(t)\right) x_{0}^{\prime}(t)+\frac{1}{2} f_{x t}^{\prime \prime}\left(t, x_{0}(t)\right)\right. \\
& \left.-\frac{1}{4} f_{x}^{\prime 2}\left(t, x_{0}(t)\right)\right] u(t)=0,
\end{aligned}
$$

with the Floquet multiplier $\beta=\alpha \exp \left[(1 / 2) \int_{0}^{T} f_{x}^{\prime}(s\right.$, $\left.\left.x_{0}(s)\right) d s\right]$.

Claim. There exists some $\xi \in[0, T]$ such that

$$
y(\xi)=0 .
$$

Assume, by way of contradiction, that (31) does not hold. Then $u(t) \neq 0$ for all $t \in[0, T]$. Dividing (30) by $u(t)$ and integrating from 0 to $T$ by parts, noticing that $u^{\prime}(0) / u(0)=$ $u^{\prime}(T) / u(T)$, we obtain

$$
\begin{gathered}
\int_{0}^{T}\left(\frac{u^{\prime}(t)}{u(t)}\right)^{2} d t+\int_{0}^{T}\left[c+\frac{1}{2} f_{x x}^{\prime \prime}\left(t, x_{0}(t)\right) x_{0}^{\prime}(t)\right. \\
\left.+\frac{1}{2} f_{x t}^{\prime \prime}\left(t, x_{0}(t)\right)-\frac{1}{4} f_{x}^{\prime 2}\left(t, x_{0}(t)\right)\right] d t=0
\end{gathered}
$$

which contradicts condition (H4). This implies that the claim (31) holds.

Since $y(\xi+T)=\alpha y(\xi)=0$, we see that $u(t)$ is a nontrivial solution of the Dirichlet boundary value problem

$$
\begin{aligned}
u^{\prime \prime}(t)+q(t) u(t) & =0, \\
u(\xi) & =u(\xi+T)=0,
\end{aligned}
$$

$$
\operatorname{sgn} u^{\prime}(\xi)=\operatorname{sgn} u^{\prime}(\xi+T),
$$


where $q(t)=\left[c+(1 / 2) f_{x x}^{\prime \prime}\left(t, x_{0}(t)\right) x_{0}^{\prime}(t)+(1 / 2) f_{x t}^{\prime \prime}\left(t, x_{0}(t)\right)-\right.$ $\left.(1 / 4) f_{x}^{\prime 2}\left(t, x_{0}(t)\right)\right]$. Multiplying (33) by $u(t)$ and integrating from $\xi$ and $\xi+T$, we have, by (H5)

$$
\begin{aligned}
& \int_{\xi}^{\xi+T} u^{\prime}(t)^{2} d t=\int_{\xi}^{\xi+T}\left[c+\frac{1}{2} f_{x x}^{\prime \prime}\left(t, x_{0}(t)\right) x_{0}^{\prime}(t)\right. \\
& \left.+\frac{1}{2} f_{x t}^{\prime \prime}\left(t, x_{0}(t)\right)-\frac{1}{4} f_{x}^{\prime 2}\left(t, x_{0}(t)\right)\right] u^{2}(t) d t \\
& \quad<\frac{[2(n+1)]^{2}}{T^{2}} \int_{\xi}^{\xi+T} u^{2}(t) d t,
\end{aligned}
$$

which contradicts the poincaré inequality.

Similarly, consider the following Dirichlet boundary value problem:

$$
\begin{aligned}
& x^{\prime \prime}(t)+q(t) x(t)=0 ; \\
& x(\xi)=x(\xi+T)=0, \\
& \operatorname{sgn} x^{\prime}(\xi)=-\operatorname{sgn} x^{\prime}(\xi+T),
\end{aligned}
$$

we can derive the following lemma.

Lemma 12 (see [9]). Let $q(t) \in L^{\infty}(0, T)$ such that

$$
\frac{[(2 n-1) \pi]^{2}}{T^{2}} \ll q(t) \ll \frac{[(2 n+1) \pi]^{2}}{T^{2}}
$$

for some $n \in \mathbb{N}$. Then (35) does not admit any nontrivial solution.

Lemma 13. Suppose that there is an integer $n \in \mathbb{N}$ such that

$$
\begin{aligned}
& \frac{1}{2} f_{t x}^{\prime \prime}(t, x(t))+\frac{1}{2} f_{x x}^{\prime \prime}(t, x(t)) x^{\prime}(t)-\frac{1}{4} f_{x}^{\prime 2}(t, x(t)) \\
& \quad+c \gg \frac{[(2 n-1) \pi]^{2}}{T^{2}}, \\
& \frac{1}{2} f_{t x}^{\prime \prime}(t, x(t))+\frac{1}{2} f_{x x}^{\prime \prime}(t, x(t)) x^{\prime}(t)-\frac{1}{4} f_{x}^{\prime 2}(t, x(t)) \\
& \quad+c \ll \frac{[(2 n+1) \pi]^{2}}{T^{2}} .
\end{aligned}
$$

Then (28) does not admit any negative Floquet multipliers. In particular, (28) does not admit any nontrivial subharmonic periodic solution of order 2.

Combining Lemmas 11 and 13, under the condition of Theorem 5, we can prove that (1) does not admit any real Floquet multiplier.

\section{Proof of Main Results}

Now we are ready to prove our main results.

\subsection{Proof of Theorem 1}

Proof. Without loss of generality, we may assume that $g(0)=$ 0 ; otherwise, we can subtract $g(0)$ from both sides of (1).
Consider the parametrized equation,

$$
\begin{aligned}
x^{\prime \prime} & (t)+\lambda \frac{d}{d t} f(t, x(t))+(1-\lambda) x^{\prime}(t)+g(x(t)) \\
& =h_{\lambda}(t)
\end{aligned}
$$

where $h_{\lambda}(t)=\lambda h(t)+(1-\lambda)(1 / T) \int_{0}^{T} h(t) d t$.

First, we claim that there is an $R>0$ which is independent of $\lambda \in[0,1]$ such that $\|x\|_{\infty}<R$ for any solution $x$ of $(37)$.

If there is not such an $R$, let $x_{n}$ be a sequence such that $\left\|x_{n}\right\| \rightarrow \infty$ and $\lambda_{n} \in[0,1]$, and denote by $z_{n}$ the ratio $x_{n} /$ $\left\|x_{n}\right\|$. Dividing (37) by $\left\|x_{n}\right\|$, multiplying by $\varphi(t) \in C_{T}^{2}$, and integrating from 0 to $T$ gives

$$
\begin{gathered}
\int_{0}^{T} z_{n} \varphi^{\prime \prime}-\varphi^{\prime}\left[\lambda_{n} \frac{f\left(t, x_{n}\right)}{\left\|x_{n}\right\|}+\left(1-\lambda_{n}\right) z_{n}\right] \\
+\varphi \frac{g\left(x_{n}\right)}{\left\|x_{n}\right\|} d t=\int_{0}^{T} \frac{\varphi h_{\lambda_{n}}}{\left\|x_{n}\right\|} d t .
\end{gathered}
$$

The condition of Theorem 1 implies that $\left(\lambda_{n} f\left(t, x_{n}\right)+\right.$ $\left.\left(1-\lambda_{n}\right) x_{n}\right) /\left\|x_{n}\right\|$ is bounded. It is precompact in the weak ${ }^{*}$ topology in $L^{1}[0, T]$. Thus there are subsequences such that $f\left(t, x_{n}\right) / x_{n} \rightarrow a(t)$ and $\lambda_{n} \rightarrow \lambda$. Passing to the limit in (38), we get

$$
\int_{0}^{T}\left\{z \varphi^{\prime \prime}-w(t) z \varphi^{\prime}+c \varphi z\right\} d t=0
$$

where $w(t)=\lambda a(t)+(1-\lambda)$.

If (4) holds, then $w(t)=\lambda a(t)+(1-\lambda) \gg 0$, which satisfies the condition of Lemma 7 . Since $z(t)$ is a $T$-periodic solution of

$$
z^{\prime \prime}+(w(t) z)^{\prime}+c z=0
$$

it follows from Lemma 7 that $z(t) \equiv 0$, which contradicts the fact that $\|z(t)\|=1$. This completes the boundedness of $\|x\|_{\infty}$ under condition (4).

Next, we show that $\|x\|_{\infty}$ is bounded which is independent of $\lambda \in[0,1]$ under condition (5).

If $\lambda<1$, then $z$ satisfies

$$
z^{\prime \prime}+(1-\lambda) z^{\prime}+c z=0 .
$$

It follows from Lemma 7 that $z(t) \equiv 0$, which contradicts $\|z(t)\|=1$.

If $\lambda=1$, then $z$ satisfies

$$
z^{\prime \prime}+c z=0
$$

By assumption, $c$ is not the eigenvalue of $L x=-x^{\prime \prime}$. Obviously, $z(t) \equiv 0$; we reach a contradiction. This shows that the solution of (1) is bounded.

Evidently, the periodic solution of (37) is equivalent to the planar system

$$
\begin{aligned}
& x^{\prime}=y-\lambda f(t, x)-(1-\lambda) x, \\
& y^{\prime}=h_{\lambda}(t)-g(x) .
\end{aligned}
$$


A natural choice for the homotopy in applying Lemma 6 is to take

$$
\begin{aligned}
& h(t, x, y, \lambda)=\left\{y-\lambda f(t, x)-(1-\lambda) x, h_{\lambda}(t)\right. \\
& \quad-\lambda g(x)-(1-\lambda) c x\} .
\end{aligned}
$$

Let $(x(t), y(t))$ be the $T$-periodic solution of (43); in order to apply Continuation Theorem to (43), we have to show that $y(t)$ is bounded. Directly from the first equation of (43) and the periodic condition, we see that there is $\tau \in[0, T]$ such that $x^{\prime}(\tau)=0$ which implies that $y(\tau)$ is bounded which is independent of $\lambda$. Integrating the second equation of (43) yields

$$
y(t)=y(\tau)+\int_{\tau}^{t}\left[h_{\lambda}(s)-g(x)\right] d s,
$$

which is bounded.

Let $r_{1}$ and $r_{2}$ be sufficiently large, and set

$$
\Omega=\left\{(x, y) \mid\|x\| \leq r_{1},\|y\| \leq r_{2}\right\} .
$$

It follows from the estimates obtained above that the equivalent planar system defined in (43)

$$
x^{\prime}=h(t, x, y, \lambda)
$$

has no solutions on $\partial \Omega$ for $\lambda \in[0,1]$. Since

$$
h(t, x, y, 0)=\{y-x, \bar{h}-c x\}=G(x, y),
$$

then condition (2) in Lemma 6 reduces to

$$
\operatorname{deg}\left(G, \Omega \cap \mathbb{R}^{2}, 0\right)=\operatorname{sgn} c \neq 0 .
$$

By applying Lemma 6, we see that (1) has at least one $T$-periodic solution.

\subsection{Proof of Theorem 2}

Proof. The idea of the proof of Theorem 2 is essentially the same as above, so here we just outline the proof and explain how to use the resonance conditions and (8)-(9) to get desired a priori estimates.

Consider the parametrized equation

$$
\begin{aligned}
& x^{\prime \prime}(t)+\lambda \frac{d}{d t} f(t, x(t))+(1-\lambda) x^{\prime}(t)+g(t, x(t)) \\
& =\lambda h(t) .
\end{aligned}
$$

First, we will show that there is $R>0$ which is independent of $\lambda \in[0,1]$ such that $\|x\|_{\infty}<R$ for any solutions $x$ of (50).

If not, let $x_{n}$ be a sequence of $T$-periodic solutions such that $\left\|x_{n}\right\| \rightarrow \infty$ and $\lambda_{n} \in[0,1]$ be the corresponding sequence. Let $z_{n}=x_{n} /\left\|x_{n}\right\|$. Noting the resonance condition (6), the same procedure of the proof of Theorem 1 gives that there exists some subsequences such that $z_{n}(t) \rightarrow z(t)$ weakly and $\lambda_{n} \rightarrow \lambda$. Passing to the limit, one obtains that $z(t)$ satisfies

$$
\int_{0}^{T}\left[z \varphi^{\prime \prime}-(1-\lambda) z \varphi^{\prime}+\left(\frac{2 n \pi}{T}\right)^{2} \varphi z\right] d t=0 .
$$

Namely,

$$
z^{\prime \prime}+(1-\lambda) z^{\prime}+\left(\frac{2 n \pi}{T}\right)^{2} z=0
$$

If $\lambda<1$, it follows from Lemma 7 that $z(t) \equiv 0$, which contradicts $\|z(t)\|=1$. Hence it remains to focus on the case that $\lambda_{n} \rightarrow 1$ and $z \in[\sin (2 n \pi / T) t, \cos (2 n \pi / T) t]$.

In $W_{T}^{1,2}$, we introduce the following symbol:

$$
\begin{aligned}
x(t) & =a_{0}+\sum_{k=1}^{\infty}\left[a_{k} \sin \frac{2 k \pi}{T} t+b_{k} \cos \frac{2 k \pi}{T} t\right], \\
x^{0}(t) & =a_{k} \sin \frac{2 k \pi}{T} t+b_{k} \cos \frac{2 k \pi}{T} t, \\
x^{\perp}(t) & =x(t)-x^{0}(t) .
\end{aligned}
$$

Since $z_{n} \rightarrow z(t) \in[\sin (2 n \pi / T) t, \cos (2 n \pi / T) t]$, we have that $\left(x_{n}^{0}+x_{n}^{\perp}\right) /\left\|x_{n}\right\| \rightarrow z(t)$.

That is to say, $\left\|x_{n}^{0}\right\| /\left\|x_{n}\right\| \rightarrow 1$ and $\left\|x_{n}^{\perp}\right\| /\left\|x_{n}\right\| \rightarrow 0$. Then $\left\|x_{n}^{\perp} / x_{n}^{0}\right\| \rightarrow 0$. Thus, the sign of $x_{n}$ is the same as that of $z$ for $n$ large enough. Taking inner product of (50) with $z$ and noting that $g(t, x)=(2 n \pi / T)^{2}+q(t, x)$, where $\lambda$ is replaced by $\lambda_{n}$ and $x$ by $x_{n}$, we have

$$
\begin{aligned}
\lambda_{n} \int_{0}^{T} h z d t= & -\int_{0}^{T} \lambda_{n} f\left(t, x_{n}^{0}+x_{n}^{\perp}\right) z^{\prime} d t \\
& +\int_{0}^{T} q\left(t, x_{n}^{0}+x_{n}^{\perp}\right) z d t .
\end{aligned}
$$

Taking the limits in the above equation, we obtain

$$
\begin{gathered}
\int_{0}^{T} h z d t \leq \liminf _{n \rightarrow \infty}\left[-\int_{0}^{T} f\left(t, x_{n}^{0}+x_{n}^{\perp}\right) z^{\prime} d t\right. \\
\left.+\int_{0}^{T} q\left(t, x_{n}^{0}+x_{n}^{\perp}\right) z d t\right] \leq-\left[\int_{z>0} a^{\prime} z d t\right. \\
\left.+\int_{z<0} b^{\prime} z d t\right]+\liminf _{n \rightarrow \infty} \int_{z>0} q\left(t, x_{n}\right) z d t \\
+\liminf _{n \rightarrow \infty} \int_{z<0} q\left(t, x_{n}\right) z d t \leq-\left[\int_{z>0} a^{\prime} z d t\right. \\
\left.+\int_{z<0} b^{\prime} z d t\right]+\int_{z>0} \alpha_{+} z d t+\int_{z<0} \beta_{-} z d t,
\end{gathered}
$$

which contradicts (9). This shows the boundedness of the periodic solutions of (50).

Similarly, by taking lim sup one can prove that periodic solutions of (50) are bounded under condition (10).

The rest is along the same line as the proof of Theorem 1 ; we omit the detail.

\subsection{Proof of Theorem 5}

Proof. Firstly, we will show that there exists a unique $T$ periodic solution. 
The existence of $T$-periodic solution of (1) has been obtained in the proof of Theorem 1. Hence it suffices to investigate the uniqueness of (1).

Let $x(t)$ and $y(t)$ be two distinct $T$-periodic solutions of (1), and $v(t)=x(t)-y(t)$. Then $v(t)$ is a nontrivial $T$-periodic solution of the following equation:

$$
x^{\prime \prime}(t)+\frac{d}{d t}(q(t) x(t))+c x(t)=0,
$$

where $q(t)=\int_{0}^{1} f_{x}^{\prime}(x(t)+s(y(t)-x(t)), t) d s$.

We claim that, under the assumption of the theorem, there exists no nontrivial $T$-periodic solution of the linearized equation (28) associated with (1).

Supposing the opposite of the previous claim, multiplying (28) by $y^{\prime}(t)$ and integrating from 0 to $T$, we have

$$
\begin{aligned}
0 & =\int_{0}^{T} y^{\prime}(t) \\
& \cdot\left[y^{\prime \prime}(t)+\frac{d}{d t}\left(f_{x}^{\prime}(x(t), t) y(t)\right)+c y(t)\right] d t \\
& =\int_{0}^{T} y^{\prime}(t) d\left(f_{x}^{\prime}(x(t), t) y(t)\right) \\
& =\left.f_{x}^{\prime}(x(t), t) y(t) y^{\prime}(t)\right|_{0} ^{T}-\int_{0}^{T} f_{x}^{\prime}(x(t), t) y(t) \\
& \cdot y^{\prime \prime}(t) d t=-\int_{0}^{T} f_{x}^{\prime}(x(t), t) y(t) \\
& \cdot\left[-c y(t)-\frac{d}{d t}\left(f_{x}^{\prime}(x(t), t) y(t)\right)\right] d t \\
& =\int_{0}^{T} c f_{x}^{\prime}(x(t), t) y^{2}(t) d t+\int_{0}^{T} f_{x}^{\prime}(x(t), t) y(t)
\end{aligned}
$$

$$
\begin{aligned}
& \cdot \frac{d}{d t}\left(f_{x}^{\prime}(x(t), t) y(t)\right) d t=c \int_{0}^{T} f_{x}^{\prime}(x(t), t) \\
& \cdot y^{2}(t) d t
\end{aligned}
$$

which contradicts the fact that both $c$ and $f_{x}^{\prime}(x(t), t)$ are positive. In the same manner, we can demonstrate that there exists no nontrivial $2 T$-periodic solutions.

By the similar argument as above, multiplying (56) by $v^{\prime}(t)$ and integrating by parts, a contradiction will be reached directly. Therefore, $v \equiv 0$.

Secondly, we will show that the unique $T$-periodic solution is locally asymptotically stable.

For each $s$ with $0 \leq s \leq 1$, we have

$$
\begin{aligned}
& s\left[c+f_{x x}^{\prime \prime}\left(t, x_{0}(t)\right) x_{0}^{\prime}(t)+f_{x t}^{\prime \prime}\left(t, x_{0}(t)\right)\right]+(1-s) \\
& >0 .
\end{aligned}
$$

Therefore, from the above conclusion, for each $s \in[0,1]$, there exists a unique periodic solution of period $2 T$ of the linear homogeneous differential equation

$$
\begin{aligned}
u^{\prime \prime} & +f_{x}^{\prime}\left(t, x_{0}(t)\right) u^{\prime} \\
& +\left(s\left[c+f_{x x}^{\prime \prime}\left(t, x_{0}(t)\right) x_{0}^{\prime}(t)+f_{x t}^{\prime \prime}\left(t, x_{0}(t)\right)\right]\right. \\
& +(1-s)) u=0 .
\end{aligned}
$$

Under the hypothesis of theorem, we can conclude that there exists no nontrivial $2 T$-periodic solution of (59) for all $0 \leq s \leq 1$.

If

$$
P(t, s)=\left(\begin{array}{cc}
0 & 1 \\
-s\left[c+f_{x x}^{\prime \prime}\left(t, x_{0}(t)\right) x_{0}^{\prime}(t)+f_{x t}^{\prime \prime}\left(t, x_{0}(t)\right)\right]-(1-s) & -f_{x}^{\prime}\left(t, x_{0}(t)\right)
\end{array}\right),
$$

then $y=\operatorname{col}\left(y_{1}, y_{2}\right)$ is a $2 T$-periodic solution of

$$
y^{\prime}(t)=P(t, s) y(t)
$$

if and only if $y_{1}=u$ and $y_{2}=u^{\prime}$, where $u$ is a nontrivial $2 T$-periodic solution of (59). Therefore, system (61) has no nontrivial $2 T$-periodic solution for $0 \leq s \leq 1$.

Let $Y(t, s)$ denote the fundamental matrix associated with system (61) for $0 \leq s \leq 1$; we have

$$
\begin{aligned}
& Y^{\prime}(t, s)=P(t, s) Y(t, s), \\
& Y(0, s)=I,
\end{aligned}
$$

where $I$ is the $2 \times 2$ identity.

If for some $v \neq 0$

$$
Y(T, s) v=-v
$$

then $y(t) \equiv Y(t, s) v$ is a solution of system (61) which satisfies $y(t)=-y(0)$. So by the fact that $-y(t)$ and $y(t+T)$ are both solutions of (61) which are equal at $t=0$ and the uniqueness theorem, it follows that $y(t+T) \equiv-y(t)$. Hence, $y(t)$ is a nontrivial $2 T$-periodic solution. So $y(t) \equiv 0$, and therefore $v=0$.

It follows that -1 is not an eigenvalue of $Y(T, s)$ for all $0 \leq$ $s \leq 1$.

A similar argument as above shows that if $Y(T, s) v=v$, then $y(t) \equiv Y(t, s) v$ is a $T$-periodic solution, and hence a $2 T$ periodic solution of (61). Therefore, for $0 \leq s \leq 1,1$ is not an eigenvalue of $Y(T, s)$.

For each $s$ with $0 \leq s \leq 1$, let $\rho_{1}(s)$ and $\rho_{2}(s)$ denote the eigenvalues of $Y(T, s)$. By standard results concerning continuous dependence of solutions of differential equations on parameters and Rouche's theorem of complex analysis, the 
moduli $\left|\rho_{1}(s)\right|$ and $\left|\rho_{2}(s)\right|$ depend continuously on $s$. We will show that

$$
\left|\rho_{i}(s)\right|<1, \quad i=1,2,0 \leq s \leq 1,
$$

and since $X(T)=Y(T, 1)$, where $X(t)$ is the fundamental matrix for the linear system

$$
x^{\prime}(t)=A(t) x(t)
$$

and $A(t)$ is the matrix function

$$
\begin{aligned}
& A(t) \\
& =\left(\begin{array}{cc}
0 & 1 \\
-\left[c+f_{x x}^{\prime \prime}\left(t, x_{0}(t)\right) x_{0}^{\prime}(t)+f_{x t}^{\prime \prime}\left(t, x_{0}(t)\right)\right] & -f_{x}^{\prime}\left(t, x_{0}(t)\right)
\end{array}\right) .
\end{aligned}
$$

Since the trace of $P(t, s)$ is equal to $-f_{x}^{\prime}\left(t, x_{0}(t)\right)$, applying Liouville's theorem, we have

$$
\rho_{1}(s) \rho_{2}(s)=e^{\int_{0}^{T} \operatorname{trace}(P(t, s)) d t}=e^{-\int_{0}^{T} f_{x}^{\prime}\left(x_{0}(t), t\right) d t}<1 .
$$

For $s=0, P(t, s)$ is equal to the matrix

$$
L=\left(\begin{array}{cc}
0 & 1 \\
-1 & -f_{x}^{\prime}\left(x_{0}(t), t\right)
\end{array}\right)
$$

so $Y(t, 0)=e^{L t}$ and $\rho_{i}(0)=e^{\lambda_{i} T}, i=1,2$, where $\lambda_{1}$ and $\lambda_{2}$ are the eigenvalues of $L$. Since $f_{x}^{\prime}\left(t, x_{0}(t)\right)>0$, it is easy to verify that both $\lambda_{1}$ and $\lambda_{2}$ have negative real parts. Therefore, $\left|\rho_{i}(0)\right|<1$ for $i=1,2$.

If (64) were not true for $0 \leq s \leq 1$, then, by continuity, there exists a $\bar{s}$ with $0<\bar{s} \leq 1$ such that $\left|\rho_{i}(\bar{s})\right|=1$, for either $i=1$ or $i=2$.

$\rho_{1}(\bar{s})$ and $\rho_{2}(\bar{s})$ can not be complex conjugates, for this means $\left|\rho_{1}(\bar{s})\right|=\left|\rho_{2}(\bar{s})\right|=1$, which contradicts with (67). Therefore, both $\rho_{1}(\bar{s})$ and $\rho_{2}(\bar{s})$ would be real, so $\rho_{i}(\bar{s})= \pm 1$ for either $i=1$ or $i=2$. This contradicts the fact that $Y(T, s)$ can not have \pm 1 as an eigenvalue for all $s \in[0,1]$.

Hence, the eigenvalues of $Y(T, s)$ have moduli less than 1 for $0 \leq s \leq 1$. In other words, the unique $T$-periodic solution is locally asymptotically stable.

Thirdly, we will show that the unique $T$-periodic solution is globally asymptotically stable.

We choose as a Lyapunov function

$$
\begin{aligned}
V= & {\left[\left(x^{\prime}(t)+f(x(t), t)\right)-\left(\phi^{\prime}(t)+f(\phi(t), t)\right)\right]^{2} } \\
& +c(x(t)-\phi(t))^{2},
\end{aligned}
$$

where $\phi(t)$ is the unique $T$-periodic solution satisfying the following equation:

$$
x^{\prime \prime}(t)+\frac{d}{d t} f(x(t), t)+c x(t)=h(t)
$$

A direct computation, by $(\mathrm{H} 1)$, yields

$$
\begin{aligned}
& \frac{d V}{d t}=2\left[x^{\prime}(t)-\phi^{\prime}(t)+f(x(t), t)-f(\phi(t), t)\right] \\
& \cdot\left[x^{\prime \prime}(t)-\phi^{\prime \prime}(t)+\frac{d}{d t}(f(x(t), t)-f(\phi(t), t))\right] \\
& +2 c[x(t)-\phi(t)]\left[x^{\prime}(t)-\phi^{\prime}(t)\right] \\
& =-2 c[x(t)-\phi(t)] \\
& \cdot\left[x^{\prime}(t)-\phi^{\prime}(t)+f(x(t), t)-f(\phi(t), t)\right] \\
& +2 c[x(t)-\phi(t)]\left[x^{\prime}(t)-\phi^{\prime}(t)\right] \\
& =-2 c[x(t)-\phi(t)]^{2} \\
& \cdot \int_{0}^{1} f_{x}^{\prime}(x(t)+s(\phi(t)-x(t)), t) d s<0 .
\end{aligned}
$$

Thus we display that the periodic solution is globally asymptotically stable.

Finally, we are in a position to prove the rate of decay of the solution to the unique $T$-periodic solution is $(1 / 2) \int_{0}^{T} f_{x}^{\prime}\left(t, x_{0}(t)\right) d t$

Consider the planar system associated with (1),

$$
\begin{aligned}
& x^{\prime}=y-f(x, t), \\
& y^{\prime}=h(t)-c x .
\end{aligned}
$$

Let $X_{0}(t)=\left(x_{0}(t), y_{0}(t)\right)$ be the unique $T$-periodic solution determined by the initial condition $X_{0}(0)=\left(x_{0}, y_{0}\right)$. Then $X_{0}$ corresponds to the unique fixed point of the poincaré mapping $P X=U(T, X)$, where $U(t, X)$ is the initial value solution of $(72)$ with $U(0, X)=X$.

Let $M(t)$ be the fundamental matrix solution of the linearization (61) of (72), and by the differentiability of $X(t)$ with respect to the initial value, the poincaré mapping can be expressed in terms of the initial value $X$ by

$$
P X-X_{0}=M(T)\left(X-X_{0}\right)+o\left(X-X_{0}\right) .
$$

By virtue of Lemmas 11 and 13, we see that $M(T)$ has a pair of conjugate eigenvalue $\rho_{1}$ and $\rho_{2}$. Thus $P(X)$ is a contracting mapping. According to Lemma 9 , there is a $C^{1}$ diffeomorphism $\phi$ which is near enough to the identity that $P X-X_{0}$ is conjugate equivalent to $M(T)$. There is an invertible constant matrix $B$ such that

$$
B^{-1} M(T) B=\left(\begin{array}{cc}
\rho_{1} & o \\
0 & \rho_{2}
\end{array}\right)=D,
$$

and we may suppose that

$$
\frac{1}{2}\left|X-X_{0}\right|<\left|\phi(X)-\phi\left(X_{0}\right)\right|<2\left|X-X_{0}\right|,
$$

for $X-X_{0}$ small, since $\phi$ is near the identity. 
So, the Lyapunov exponent is given by

$$
\begin{aligned}
\mu= & \lim _{n \rightarrow \infty} \frac{1}{n T} \ln \left|P^{n} X-X_{0}\right|=\lim _{n \rightarrow \infty} \frac{1}{n T} \\
& \cdot \ln \left|\phi \circ M(T)^{n} \circ \phi^{-1}(X)-\phi \circ M(T)^{n} \circ \phi^{-1}\left(X_{0}\right)\right| \\
& =\lim _{n \rightarrow \infty} \frac{1}{n T} \ln \left|D^{n} \circ B^{-1}\left[\phi^{-1}(X)-\phi^{-1}\left(X_{0}\right)\right]\right| \\
& =-\frac{1}{2} \int_{0}^{T} f_{x}^{\prime}\left(t, x_{0}(t)\right) d t .
\end{aligned}
$$

Hence, the rate of decay of the solution to the unique $T$-periodic solution is $(1 / 2) \int_{0}^{T} f_{x}^{\prime}\left(t, x_{0}(t)\right) d t$, independent of the initial value $X$. This completes the proof of Theorem 5 .

\section{Conflicts of Interest}

The authors declare that there are no conflicts of interest regarding the publication of this paper.

\section{References}

[1] C. Fabry, J. Mawhin, and M. N. Nkashama, "A multiplicity result for periodic solutions of forced nonlinear second order ordinary differential equations," Bulletin of the London Mathematical Society, vol. 18, no. 2, pp. 173-180, 1986.

[2] J.-P. Gossez and P. Omari, "Periodic solutions of a second order ordinary differential equation: a necessary and sufficient condition for nonresonance," Journal of Differential Equations, vol. 94, no. 1, pp. 67-82, 1991.

[3] C. P. Gupta, J. J. Nieto, and L. Sanchez, "Periodic solutions of some Liénard and Duffing equations," Journal of Mathematical Analysis and Applications, vol. 140, no. 1, pp. 67-82, 1989.

[4] A. Zitan and R. Ortega, "Existence of asymptotically stable periodic solutions of a forced equation of Liénard type," Nonlinear Analysis. Theory, Methods \& Applications. An International Multidisciplinary Journal, vol. 22, no. 8, pp. 993-1003, 1994.

[5] A. Fonda and P. Habets, "Periodic solutions of asymptotically positively homogeneous differential equations," Journal of Differential Equations, vol. 81, no. 1, pp. 68-97, 1989.

[6] D. Qian, "Periodic solutions of Liénard equations with superlinear asymmetric nonlinearities," Nonlinear Analysis. Theory, Methods \& Applications. An International Multidisciplinary Journal, vol. 43, no. 5, Ser. A: Theory Methods, pp. 637-654, 2001.

[7] A. C. Lazer and P. J. Mckenna, "On the existence of stable periodic solutions of differential equations of Duffing type," Proceedings of the American Mathematical Society, vol. 110, no. 1, pp. 125-133, 1990.

[8] H. Chen and Y. Li, "Rate of decay of stable periodic solutions of Duffing equations," Journal of Differential Equations, vol. 236, no. 2, pp. 493-503, 2007.

[9] H. Chen and Y. Li, "Bifurcation and stability of periodic solutions of Duffing equations," Nonlinearity, vol. 21, no. 11, pp. 2485-2503, 2008.

[10] J. Mawhin, “Topological degree and boundary value problems for nonlinear differential equations," in Topological Methods for Ordinary Differential Equations, vol. 1537 of Lecture Notes in Mathematics, pp. 74-142, Springer, Berlin, Germany, 1993.
[11] J. L. Massera, "The existence of periodic solutions of systems of differential equations," Duke Mathematical Journal, vol. 17, pp. 457-475, 1950.

[12] M. C. Irwin, Smooth dynamical systems, vol. 94 of Pure and Applied Mathematics, Academic Press, Inc. [Harcourt Brace Jovanovich, Publishers], New York-London, 1980. 


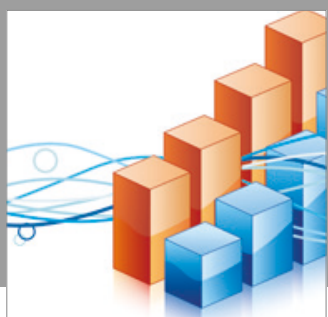

Advances in

Operations Research

vatersals

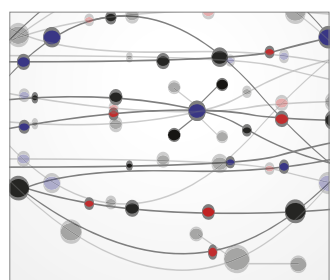

\section{The Scientific} World Journal
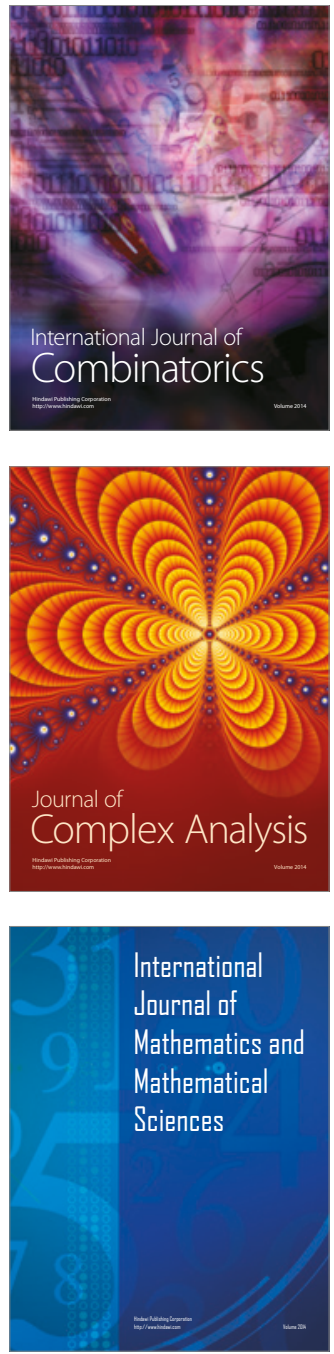
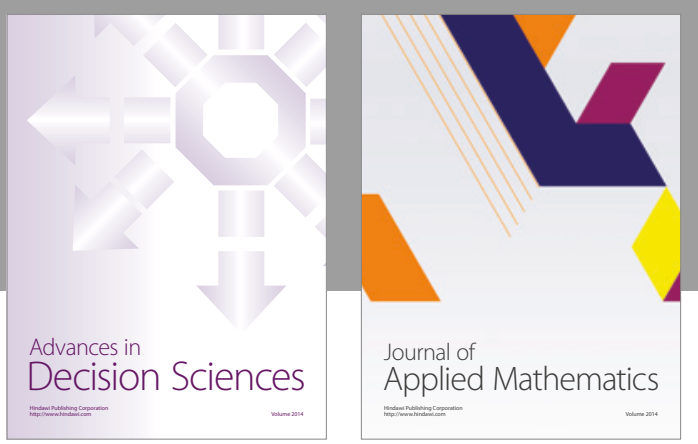

Algebra

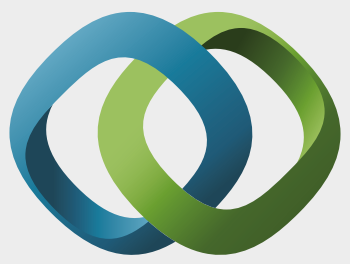

\section{Hindawi}

Submit your manuscripts at

https://www.hindawi.com
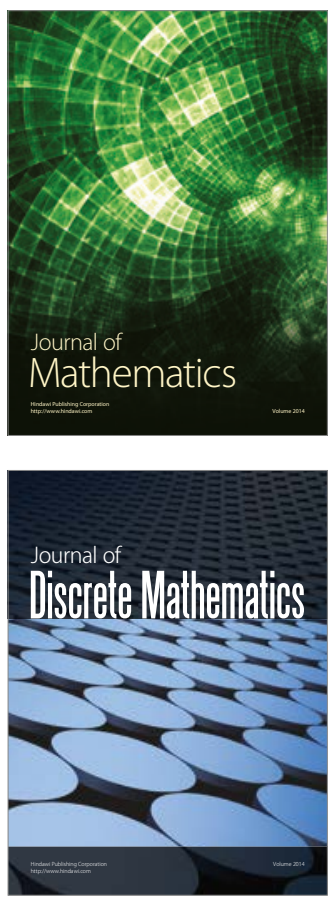

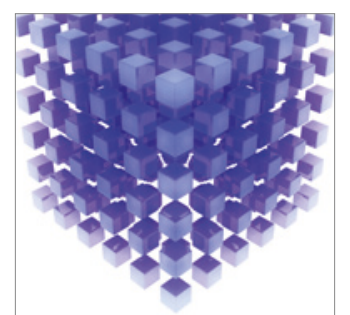

Mathematical Problems in Engineering
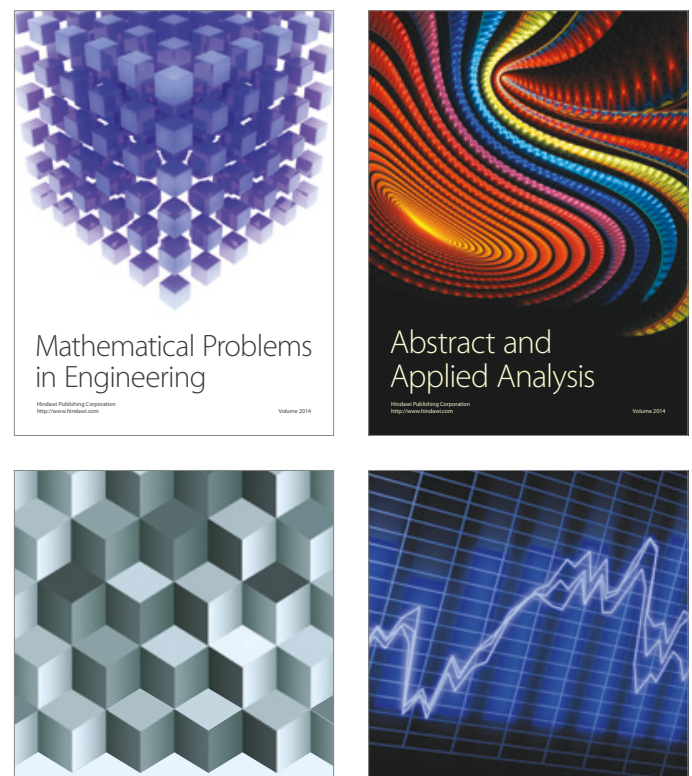

Journal of

Function Spaces

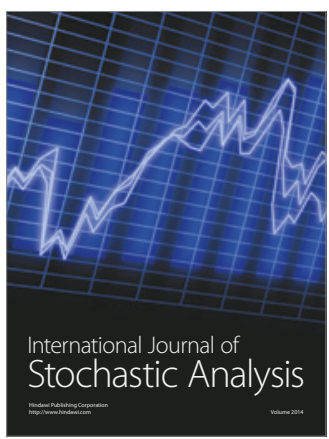

Probability and Statistics
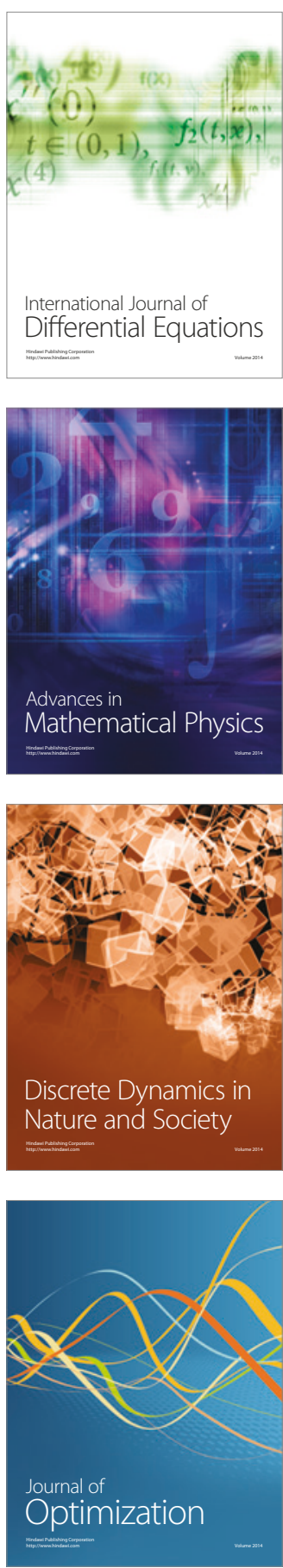